\title{
Progress in the determination of some astronomical constants from radiometric observations of planets and spacecraft
}

\author{
E. V. Pitjeva \\ Institute of Applied Astronomy of Russian Academy of Sciences, Kutuzov Quay 10, 191187 St Petersburg, Russia \\ Received 14 February 2001 / Accepted 13 March 2001

\begin{abstract}
Modern radiometric observations of planets, beginning in 1961, make it possible to determine and improve a broad set of astronomical constants from the value of the astronomical unit (AU) to parameters of PPN formalism. Three main factors that influence the progress in the determination of astronomical constants 1) reductions of the observational data, 2) dynamical models of planet motion, 3) observational data themselves - are demonstrated in this paper. The reduction of the measurements included all relevant corrections, including the modeling of the topography of Mercury and Venus which reduced the rms residuals for observations by $14.5 \%$ and $23 \%$ correspondingly. The formal standard deviations of the solution elements of the planets and the AU are improved by 30-50\% using the DE405 or EPM2000 ephemerides constructed in IAA (Russia) instead of DE200. It was shown that including the measurements of the Viking and Pathfinder landers, being free from the uncertainties due to planetary topography, into the observational data reduces the uncertainties of adjusted parameters by 1-2 orders. The astronomical constants obtained in the fitting process of the DE405 and EPM2000 ephemerides to data totaling more 80000 radiometric observations of planets and spacecraft are given.
\end{abstract}

Key words. astrometry - ephemerides - solar system: minor planets - planets and satellites

\section{Reductions of observations}

Radiometric observations of planets began in 1961 with radar-ranging, and since then many additional techniques have come widely into astronomical practice, making it possible to determine with high accuracy various astronomical constants.

Reductions of modern observations, including relativistic corrections, the Shapiro time-delay effect near the Sun, the transition from the coordinate time of the ephemerides to the proper time of the observer, and the effects of propagation of electromagnetic signals in the Earth troposphere and in the solar corona, are well known and are described, for example, by Standish (1990).

The reduction for the topography of the radarobservable planets causes some difficulty. Large variations in the surface of a planet bring in significant systematic errors (up to $20 \mathrm{~km}$ for Mars), cause a large noise component of the time delay, and therefore worsen the accuracy of the determination of parameters. The correction of observations of Venus and Mars for their topography has been carried out with the help of modern hypsometric maps of the surfaces of these planets, and also by the representation of the global topography with an expansion of spherical functions of 16-18 degrees. After taking into

Send offprint requests to: E. V. Pitjeva,

e-mail: evp@quasar.ipa.nw.ru account the topography of Venus and Mars, the systematic component of the residuals has been significantly removed and the rms residuals for the Venus observations has been decreased by $23 \%$. Details of the topography reduction can be found in Pitjeva (1996).

The global topography of Mercury is virtually unknown, although some works based on Mercury radar altimetry have been announced (for example, Harmon et al. 1985). The topography of Mercury has been represented, as in the papers by Anderson et al. (1996) and Standish (1998), by Legendre functions to the second order. The expansion coefficients have been estimated from the 297 available ranging observations of Mercury obtained during 1966-1997, having a priori uncertainties better than $2 \mathrm{~km}$ (Pitjeva 2000). The adjustment to the Mercury surface has decreased the rms residuals for Mercury observations by $14.5 \%$.

\section{Dynamic models of planetary motion}

The influence of dynamical models on the determination of parameters has been revealed by the use of various planetary ephemerides as the bases for the adjustment to the same set of observations. The JPL ephemerides - DE200 (Standish 1990), DE403 (Standish et al. 1995), DE405 (Standish 1998) and the similar ephemerides EPM98, EPM2000 (Pitjeva 2001), constructed at the Institute of 
Applied Astronomy of Russian Academy of Sciences, have been used with the same set of observational data.

Common to all these dynamical models is a simultaneous numerical integration of the equations of motion of the nine planets, the Sun, the Moon and lunar physical libration, performed in the Parameterized Post-Newtonian metric for the harmonic coordinates $(\alpha=0)$ and General Relativity values $(\beta=\gamma=1)$. The Ephemerides of Planets and the Moon (EPM2000) took into account the perturbations from 300 asteroids by numerical integration of the equations of motion of all objects, but including the mutual perturbations of only the planets, moon, sun, and the 5 most significant asteroids. Previously, EPM98 had accounted for the asteroid perturbations in a manner similar to that used for DE403.

There are slight differences among the models of the various ephemerides: the modelling of the lunar libration is different, but this has no significant effect on the parameters considered in this paper; the solar oblateness $J_{2}=2.010^{-7}$, obtained from some astrophysical estimations (Duvall et al. 1984; Brown 1989), has been taken into account; and the constructed ephemerides differ only slightly by the accuracy of interpolation for planetary coordinates by a set of Chebyshev polynomials. DE200 differs significantly from the other ephemerides in the modelling of the perturbations from asteroids: only the three most massive asteroids were taken into account in DE200, while the other ephemerides were supplemented with the modelling of perturbations from 300 asteroids upon the orbits of the planets.

The astronomical parameters have been determined by computing for each ephemeris residuals of the same observational data and then by re-computing them by adjusting a number of ephemeris parameters. The data included radar ranging of Mercury and Venus and spacecraft ranging and doppler of the Viking and Pathfinder martian landers (observations marked by a $*$ in Table 2 ). Note that the uncertainties, given in this paper, are formal standard deviations; realistic error bounds may be an order of magnitude larger. The formal standard deviations are given in Table 1 for the orbital elements of Mercury, Venus, Earth and Mars: $a_{i}, \sin i_{i} \cos \Omega_{i}, \sin i_{i} \sin \Omega_{i}, e_{i} \cos \pi_{i}$, $e_{i} \sin \pi_{i}, \lambda_{i}$, where $a$ - the semi-major axis, $i$ - the inclination of the orbit, $\Omega$ - the ascending node, $e$ - the eccentricity, $\pi$ - the longitude of perihelion, $\lambda$ - the mean longitude, index ${ }_{i}=1,2,3,4$ - planets; also given in Table 1 are the corrections to the value of the astronomical unit $(\mathrm{AU}=149597870691.0 \mathrm{~m})$ and to the Mars precession $\left(\dot{\Omega}_{q}=-7^{\prime \prime} .576 / \mathrm{y}\right)$ with their uncertainties determined by using the various ephemerides.

From Table 1 it is seen that, first, the dynamical models of the ephemerides DE403, DE405, EPM98, EPM2000 are nearly equivalent and yield virtually identical accuracy for the determination of the parameters. Secondly, the formal standard deviations of the solution parameters are improved by $30-50 \%$ using DE403, DE405, EPM98, EPM2000 ephemerides instead of DE200.
In addition, it should be noted that the DE200 ephemerides were created before many spacecraft-based determinations of the relevant parameters existed. Thus, many of the fitting parameters (e.g., planet masses, station locations) were less accurately determined for DE200 than they are known today. Consequently, part of the higher uncertainties of DE200 must be attributed to the embedded effect of these parameters. The numerical experiments have shown that the accuracy of adjusted parameters essentially depended on values of planet masses and asteroid modeling.

\section{Observational data}

The quality and quantity of the observational data affect the accuracy of estimated parameters. The accuracy of the first observations of time delay $(\tau)$ of planets, obtained in 1961-1962 was 200-500 $\mu \mathrm{s}(30-80 \mathrm{~km})$; the accuracy of 1964-1969 ranging observations improved to $30 \mu \mathrm{s}(5 \mathrm{~km})$; the accuracy of modern ranging observations has achieved $0.5 \mu \mathrm{s}(80 \mathrm{~m})$. Unfortunately, such observations are corrupted by the peculiarities of the planetary relief, and some uncertainties remain in planetary ranging in spite of the corrections for topography. Therefore, the extremely precise observations of the martian Viking and Pathfinder landers, being free from the uncertainties due to the planetary topography, are of great importance.

Measurements of time delay $\tau$ of the Viking martian landers were obtained during 1976-1982. For 20 years these observations were the most accurate of all planetary position data. Observations of the new martian Pathfinder lander have now been obtained during three months of 1997. Simultaneously the observations of differenced range $d \tau$ were carried out. Similar differenced range data have been recovered from 1976-1978 measurements by the Viking-1 lander.

The observations of the martian landers have permitted the highly accurate improvement of not only the orbital elements of Earth and Mars, but also the parameters of Mars rotation; in particular, such an important parameter for understanding of Mars geophysics as the mean precession rate of Mars (Yoder \& Standish 1997; Folkner et al. 1997; Pitjeva 1999).

Since the orbit of Mars is perturbed significantly by Jupiter and a number of asteroids, the observations of the martian landers contain information concerning the mass of Jupiter, elements of its orbit, as well as about the masses of the three largest asteroids: Ceres, Pallas and Vesta (Standish \& Hellings 1989; Pitjeva 1997).

An electronic date base of planetary observational data used in the production of ephemerides has been created on the INTERNET: "http://ssd.jpl.nasa.gov/iau-comm4". All ranging observations from this data base together with Russian ranging measurements of planets obtained during 19621995 have been used in this paper. Thus we have over 80000 radiometric observations of the inner planets during 36 years. For this paper, all Mars observations 
Table 1. The formal standard deviations of parameters, readjusted to the same set of observations, using a selected set of parameters and DE200, DE403, EPM98, DE405, EPM2000

\begin{tabular}{lccccc}
\hline parameters & DE200 & DE403 & EPM98 & DE405 & EPM2000 \\
\hline$a_{1}[\mathrm{~m}]$ & 0.430 & 0.306 & 0.309 & 0.305 & 0.308 \\
$\sin i_{1} \cos \Omega_{1}[\mathrm{mas}]$ & 5.451 & 3.886 & 3.917 & 3.874 & 3.909 \\
$\sin i_{1} \sin \Omega_{1}[\mathrm{mas}]$ & 6.010 & 4.284 & 4.318 & 4.272 & 4.310 \\
$e_{1} \cos \pi_{1}[\mathrm{mas}]$ & 0.537 & 0.383 & 0.386 & 0.382 & 0.385 \\
$e_{1} \sin \pi_{1}[\mathrm{mas}]$ & 0.460 & 0.328 & 0.331 & 0.327 & 0.330 \\
$\lambda_{1}[\mathrm{mas}]$ & 1.708 & 1.218 & 1.227 & 1.214 & 1.225 \\
\hline$a_{2}[\mathrm{~m}]$ & 1.463 & 1.042 & 1.050 & 1.038 & 1.048 \\
$\sin i_{2} \cos \Omega_{2}[\mathrm{mas}]$ & 2.322 & 1.655 & 1.668 & 1.650 & 1.665 \\
$\sin i_{2} \sin \Omega_{2}[\mathrm{mas}]$ & 2.244 & 1.600 & 1.612 & 1.595 & 1.609 \\
$e_{2} \cos \pi_{2}[\mathrm{mas}]$ & 0.127 & 0.090 & 0.091 & 0.090 & 0.091 \\
$e_{2} \sin \pi_{2}[\mathrm{mas}]$ & 0.119 & 0.085 & 0.086 & 0.085 & 0.085 \\
$\lambda_{2}[\mathrm{mas}]$ & 0.990 & 0.706 & 0.711 & 0.704 & 0.710 \\
\hline$a_{3}[\mathrm{~m}]$ & 0.180 & 0.109 & 0.104 & 0.103 & 0.103 \\
$e_{3} \cos \pi_{3}[\mathrm{mas}]$ & 0.001 & 0.001 & 0.001 & 0.001 & 0.001 \\
$e_{3} \sin \pi_{3}[\mathrm{mas}]$ & 0.001 & 0.001 & 0.001 & 0.001 & 0.001 \\
\hline$a_{4}[\mathrm{~m}]$ & 0.518 & 0.291 & 0.271 & 0.270 & 0.270 \\
$\sin i_{4} \cos \Omega_{4}[\mathrm{mas}]$ & 0.033 & 0.024 & 0.024 & 0.024 & 0.024 \\
$\sin i_{4} \sin \Omega_{4}[\mathrm{mas}]$ & 0.040 & 0.026 & 0.026 & 0.026 & 0.026 \\
$e_{4} \cos \pi_{4}[\mathrm{mas}]$ & 0.002 & 0.002 & 0.002 & 0.002 & 0.002 \\
$e_{4} \sin \pi_{4}[\mathrm{mas}]$ & 0.003 & 0.002 & 0.002 & 0.002 & 0.002 \\
$\lambda_{4}[\mathrm{mas}]$ & 0.013 & 0.008 & 0.008 & 0.008 & 0.008 \\
\hline $\operatorname{scale~factor}[\mathrm{m} / \mathrm{au}]$ & 21.354 & 1.408 & 0.310 & 2.010 & 0.206 \\
$\dot{\Omega}_{q}[\mathrm{mas} / \mathrm{yr}]$ & \pm 0.288 & \pm 0.190 & \pm 0.186 & \pm 0.184 & \pm 0.184 \\
& -10.98 & 2.35 & 4.61 & -3.42 & -7.36 \\
\hline & \pm 7.28 & \pm 5.17 & \pm 5.20 & \pm 5.15 & \pm 5.17 \\
\hline & & & & &
\end{tabular}

and, as a rule, Venus observations, carried out within a single day have been combined into normal points after the necessary reductions. The observations have been weighted in accordance with their a priori standard deviations. The orbital elements of Jupiter have been fitted to all measurements of spacecraft at Jupiter (Pioneer-10,11, Voyager-1,2, Ulysses, Galileo) and its VLA thermal emission, as well as to those of the martian landers, as indicated above. The observations used in this paper are described in Table 2. The columns contain the station or the observed object, the components measured, the time coverage, the number of observations and normal points, and the a priori uncertainties of measurements. Table 3 gives corrections (with their formal uncertainties) to parameters of the EPM2000 fitted to three different sets of observations:

- version I - only ranging observations of planets;

- version II - ranging observations of planets and Viking-1,2;

- version III - ranging of planets, of Viking and Pathfinder, differenced range observations of Viking and Pathfinder.

It is seen from Table 3 that including the Viking and Pathfinder landers into the observational data reduces the uncertainties of the adjusted parameters by 1-2 orders and their formal accuracy reaches the microarcsecond level.

\section{The adjusted astronomical constants}

A number of parameters have been adjusted into the fitting process of the DE405 and EPM2000 ephemerides to data totaling over 80000 radiometric observations of planets and spacecraft.

Tables 4 and 5 give the formal standard deviations of the orbital elements of Mercury, Venus, Earth, Mars, Jupiter and the parameters of Mars rotation. The solution value of the astronomical unit is in good agreement with that of DE405:

$$
\begin{aligned}
& \mathrm{AU}_{\mathrm{EPM} 2000}=(149597870691.2 \pm 0.2) \mathrm{m}, \\
& \mathrm{AU}_{\mathrm{DE} 405}=149597870691 \mathrm{~m}
\end{aligned}
$$

and is $1.5 \mathrm{~m}$ longer than its last value by Standish (2000a):

$\mathrm{AU}_{\text {Standish }}=(149597870689.7 \pm 0.4) \mathrm{m}$.

Following formulas of Folkner et al. (1997) and considering the gravity coefficient of Mars $J_{2}$, obtained from detection of the Mars gravity field with the use of the Viking orbiter and other tracking data by Konopliv \& Sjogren (1995), to 
Table 2. Radiometric observations used in the ephemerides solutions

MERCURY

\begin{tabular}{|c|c|c|c|c|c|}
\hline $\begin{array}{l}\text { station, } \\
\text { object }\end{array}$ & type & $\begin{array}{l}\text { time } \\
\text { interval }\end{array}$ & $\begin{array}{l}\text { number } \\
\text { of obs. }\end{array}$ & $\begin{array}{l}\text { normal } \\
\text { points }\end{array}$ & $\begin{array}{l}\text { a priori } \\
\text { accuracy }\end{array}$ \\
\hline Millstone & $\tau$ & 1964 & 5 & - & $50-500 \mu \mathrm{s}$ \\
\hline Haystack* & $\tau$ & 1966-1971 & 217 & - & $20 \mu \mathrm{s}$ \\
\hline Arecibo* & $\tau$ & 1964-1982 & 341 & 323 & $20-200 \mu \mathrm{s}$ \\
\hline Goldstone* & $\tau$ & $1971-1997$ & 259 & 138 & $10-20 \mu \mathrm{s}$ \\
\hline \multirow[t]{2}{*}{ Crimea } & $\tau$ & 1980-1995 & 75 & 23 & $8-32 \mu \mathrm{s}$ \\
\hline & & VENUS & & & \\
\hline Millstone* & $\tau$ & 1961-1967 & 135 & - & $10-800 \mu \mathrm{s}$ \\
\hline Haystack ${ }^{*}$ & $\tau$ & 1966-1971 & 219 & - & $10 \mu \mathrm{s}$ \\
\hline Arecibo* & $\tau$ & $1964-1970$ & 319 & - & $20-100 \mu \mathrm{s}$ \\
\hline Goldstone* & $\tau$ & 1964-1990 & 512 & - & $10-40 \mu \mathrm{s}$ \\
\hline \multirow[t]{2}{*}{ Crimea } & $\tau$ & 1962-1995 & 1139 & 170 & $1-150 \mu \mathrm{s}$ \\
\hline & & MARS & & & \\
\hline Haystack & $\tau$ & $1967-1973$ & 3801 & 133 & $0.5-80 \mu \mathrm{s}$ \\
\hline Arecibo & $\tau$ & 1965-1973 & 1680 & 43 & $0.5-300 \mu \mathrm{s}$ \\
\hline Goldstone & $\tau$ & 1969-1994 & 48989 & 149 & $0.5-4 \mu \mathrm{s}$ \\
\hline Crimea & $\tau$ & 1971-1995 & 381 & 78 & $1-32 \mu \mathrm{s}$ \\
\hline Mariner-9 & $\tau$ & $1971-1972$ & 643 & - & $0.1-1.8 \mu \mathrm{s}$ \\
\hline Viking-1* & $\tau$ & 1976-1982 & 1161 & - & $0.046-0.080 \mu \mathrm{s}$ \\
\hline Viking-1* & $\mathrm{d} \tau$ & $1976-1978$ & 14980 & - & $1.3-27 \mathrm{~mm} / \mathrm{s}$ \\
\hline Viking-2* & $\tau$ & $1976-1977$ & 80 & - & $0.046-0.067 \mu \mathrm{s}$ \\
\hline Pathfinder* & $\tau$ & 1997 & 90 & - & $0.067-0.146 \mu \mathrm{s}$ \\
\hline \multirow[t]{2}{*}{ Pathfinder* } & $\mathrm{d} \tau$ & 1997 & 7576 & - & $0.11 \mathrm{~mm} / \mathrm{s}$ \\
\hline & & JUPITER & & & \\
\hline spacecraft, VLA & $\alpha$ & 1979-1995 & - & 4 & $0 . .003-0 . .046$ \\
\hline spacecraft, VLA & $\delta$ & 1979-1995 & - & 4 & $0 .{ }^{\prime \prime} 005-0 . .2$ \\
\hline spacecraft & $\tau$ & 1973-1995 & - & 6 & $3-40 \mu \mathrm{s}$ \\
\hline spacecraft & $\alpha \delta$ & $1996-1997$ & - & 23 & $0^{\prime \prime} 007-0 .{ }^{\prime \prime} 012$ \\
\hline
\end{tabular}

Table 3. Corrections to parameters of the EPM2000, fitted to three different sets of observations

\begin{tabular}{lccc}
\hline parameters & version I & version II & version III \\
\hline$a_{3}[\mathrm{~m}]$ & $-2.438 \pm 3.565$ & $-2.155 \pm 0.201$ & $-1.877 \pm 0.084$ \\
$e_{3} \cos \pi_{3}[\mathrm{mas}]$ & $-0.372 \pm 0.095$ & $-0.006 \pm 0.002$ & $-0.012 \pm 0.001$ \\
$e_{3} \sin \pi_{3}[\mathrm{mas}]$ & $-0.346 \pm 0.098$ & $-0.005 \pm 0.002$ & $-0.005 \pm 0.001$ \\
\hline$a_{4}[\mathrm{~m}]$ & $1.860 \pm 9.640$ & $-4.323 \pm 0.587$ & $-5.063 \pm 0.224$ \\
$\sin i_{4} \cos \Omega_{4}[\mathrm{mas}]$ & $1.385 \pm 1.218$ & $-0.146 \pm 0.030$ & $-0.263 \pm 0.023$ \\
$\sin i_{4} \sin \Omega_{4}[\mathrm{mas}]$ & $-7.086 \pm 1.289$ & $-0.002 \pm 0.034$ & $0.233 \pm 0.021$ \\
$e_{4} \cos \pi_{4}[\mathrm{mas}]$ & $0.133 \pm 0.088$ & $-0.018 \pm 0.002$ & $-0.002 \pm 0.001$ \\
$e_{4} \sin \pi_{4}[\mathrm{mas}]$ & $-0.403 \pm 0.109$ & $-0.022 \pm 0.003$ & $-0.015 \pm 0.001$ \\
$\lambda_{4}[\mathrm{mas}]$ & $-2.265 \pm 0.324$ & $-0.183 \pm 0.013$ & $-0.035 \pm 0.008$ \\
\hline scale factor $[\mathrm{m} / \mathrm{au}]$ & $-307.634 \pm 69.324$ & $1.022 \pm 0.686$ & $0.710 \pm 0.188$ \\
$\dot{\Omega}_{q}[\mathrm{mas} / \mathrm{yr}]$ & - & $-672.28 \pm 134.27$ & $4.97 \pm 5.28$ \\
\hline
\end{tabular}

be equal to 0.0019586 , the corresponding normalized polar moment of inertia of Mars was determined as

$C / M R^{2}=0.3664 \pm 0.0013(5 \sigma)$ which practically coincides with the value by Folkner et al. (1997):

$C / M R^{2}=0.3662 \pm 0.0017(5 \sigma)$.

With ranging observations of high accuracy covering the time interval of over 30 years one may estimate the time 
Table 4. The formal standard deviations of elements of the planets

\begin{tabular}{llllllll}
\hline planet & theory & $a$ & $\begin{array}{l}\sin i \cos \Omega \\
{[\mathrm{mas}]}\end{array}$ & $\begin{array}{l}\sin i \sin \Omega \\
{[\mathrm{mas}]}\end{array}$ & $\begin{array}{l}e \cos \pi \\
{[\mathrm{mas}]}\end{array}$ & $\begin{array}{l}e \sin \pi \\
{[\mathrm{mas}]}\end{array}$ & $\begin{array}{l}\lambda \\
{[\mathrm{mas}]}\end{array}$ \\
\hline \multirow{2}{*}{ Mercury } & DE405 & 0.186 & 3.156 & 3.316 & 0.320 & 0.277 & 0.823 \\
& EPM2000 & 0.187 & 3.169 & 3.329 & 0.321 & 0.279 & 0.826 \\
Venus & DE405 & 0.307 & 0.604 & 0.592 & 0.039 & 0.040 & 0.183 \\
& EPM2000 & 0.309 & 0.606 & 0.594 & 0.039 & 0.040 & 0.184 \\
Earth & DE405 & 0.083 & - & - & 0.001 & 0.001 & - \\
& EPM2000 & 0.084 & - & - & 0.001 & 0.001 & - \\
\multirow{4}{*}{ Jupiter } & DE405 & 0.219 & 0.023 & 0.020 & 0.001 & 0.001 & 0.007 \\
& EPM2000 & 0.224 & 0.023 & 0.021 & 0.001 & 0.001 & 0.008 \\
& DE405 & 672.4 & 4.798 & 4.070 & 0.319 & 0.321 & 1.160 \\
& EPM2000 & 675.1 & 4.817 & 4.086 & 0.321 & 0.322 & 1.164 \\
\hline
\end{tabular}

Table 5. The parameters of Mars rotation

\begin{tabular}{llllll}
\hline theory & $\dot{V}\left[{ }^{\circ} /\right.$ day $]$ & $I_{q}\left[^{\circ}\right]$ & $\dot{I}_{q}\left[^{\prime \prime} / \mathrm{y}\right]$ & \multicolumn{1}{l}{$\Omega_{q}\left[^{\circ}\right]$} & $\dot{\Omega}_{q}\left[^{\prime \prime} / \mathrm{y}\right]$ \\
\hline \multirow{2}{*}{ DE405 } & 350.891985144 & 25.1893949 & -0.0028 & 35.437715 & -7.5741 \\
& \pm 0.000000011 & \pm 0.0000050 & \pm 0.0024 & \pm 0.000020 & \pm 0.0050 \\
\multirow{2}{*}{ EPM2000 } & 350.891985129 & 25.1893932 & -0.0036 & 35.437724 & -7.5712 \\
& \pm 0.000000011 & \pm 0.0000050 & \pm 0.0024 & \pm 0.000020 & \pm 0.0050 \\
\hline
\end{tabular}

Table 6. Parameters of PPN formalism, $\dot{G} / G$ and the solar quadrupole moment

\begin{tabular}{llcc|cl}
\hline theory & $\dot{G} / G\left(10^{-11} \mathrm{yr}^{-1}\right)$ & $\beta-1$ & $\gamma-1$ & $\Delta \dot{\pi}_{\mathrm{Mer}}\left({ }^{\prime \prime} \mathrm{cy}^{-1}\right)$ & $J_{2}\left(10^{-7}\right)$ \\
\hline DE405 & $0.001 \pm 0.008$ & $0.0001 \pm 0.0001$ & $0.0004 \pm 0.0001$ & $0.0058 \pm 0.0086$ & $2.46 \pm 0.68$ \\
EPM2000 & $0.004 \pm 0.008$ & $0.0004 \pm 0.0002$ & $0.0001 \pm 0.0001$ & $0.0055 \pm 0.0085$ & $2.43 \pm 0.67$ \\
\hline
\end{tabular}

derivative of the gravitational constant $(\dot{G} / G)$, characterizing the fundamental properties of our physical timespace, and representing a test of the PPN parameters. The results for these, given in the first three columns of Table 6, show no significant departure from their standard values.

In a second solution, we set $\beta=1, \gamma=1$, and $\dot{G} / G=0$, and then we solved for a correction to the motion of Mercury's perihelion by using the partial derivative, $\frac{\partial \tau}{\partial \dot{\pi}}=\frac{\partial \tau}{\partial \pi} *\left(t-t_{0}\right)$, where $\tau$ is the measured round-trip light-time of a ranging measurement. Then, from the linear combination (see, for example, Brumberg 1972),

$\dot{\pi}_{1}=42^{\prime \prime} 98\left[(2+2 \gamma-\beta) / 3+0.296 J_{2} 10^{4}\right]$

we determined the corrected value for $J_{2}$ as shown in the last column of Table 6 .

A test ephemeris, EPMTEST, was constructed to examine the validity of the fitting process and the estimation of the solar oblateness. The EPMTEST ephemeris differs from EPM2000 by only the zero solar oblateness (the $J_{2}=2.010^{-7}$ solar quadrupole moment is used for EPM2000). The secular trend of the Mercury perihelion and the corresponding solar quadrupole moment together with other parameters have been obtained into the fitting process of EPMTEST to all ranging observations. The resulting dynamical value of the solar quadrupole moment:

$J_{2 \text { test }}=(2.36 \pm 0.65) 10^{-7}$

agrees remarkably with the values from Table 6 and coincides on the level of an accuracy with the astrophysical value obtained by Pijpers (1998) from helioseismic measurements:

$J_{2 \text { Pijpers }}=(2.18 \pm 0.06) 10^{-7}$.

Tables 7 and 8 present estimates of the masses of Jupiter, Ceres, Pallas, Vesta and densities of the three major taxonomic asteroids classes (C, S, M) accepted for DE405, obtained by the author using the ephemerides DE405 and EPM2000 and obtained by Standish (2000a) from most recent ephemeris solution, fit to all relevant existing observational data. It is seen that the recent estimates of the masses of Ceres, Pallas, Vesta and the densities of taxonomic classes of asteroids are close to each other and agree well with the values $(1.29 \pm 0.03) \mathrm{g} / \mathrm{cm}^{3}$ for carbonaceous asteroid 253 Mathilde (Yeomans et al. 1997) and $(2.67 \pm 0.03) \mathrm{g} / \mathrm{cm}^{3}$ for asteroid Eros of S class (Yeomans et al. 1999), determined from tracking data during the NEAR flyby. 
Table 7. Sun-Jupiter and Ceres-Sun, Pallas-Sun, Vesta-Sun mass ratios

\begin{tabular}{lllll}
\hline theory, author & $M_{\odot} / M_{\text {Jup }}$ & $\begin{array}{c}M_{\mathrm{Cer}} / M_{\odot} \\
10^{-10}\end{array}$ & $\begin{array}{c}M_{\mathrm{Pal}} / M_{\odot} \\
10^{-10}\end{array}$ & $\begin{array}{c}M_{\mathrm{Ves}} / M_{\odot} \\
10^{-10}\end{array}$ \\
\hline DE405 & 1047.34860 & 4.70 & 1.00 & 1.30 \\
PitjevaDE $_{\text {Pitjeva }}$ & $1047.34856 \pm 0.00003$ & $4.71 \pm 0.01$ & $1.00 \pm 0.01$ & $1.31 \pm 0.01$ \\
Standish $_{2000}$ & $1047.34866 \pm 0.00003$ & $4.81 \pm 0.01$ & $1.00 \pm 0.01$ & $1.36 \pm 0.01$ \\
& & $4.76 \pm 0.01$ & $1.08 \pm 0.01$ & $1.35 \pm 0.01$ \\
\hline
\end{tabular}

Table 8. The densities of the $\mathrm{C}, \mathrm{S}, \mathrm{M}$ taxonomic asteroid classes (in $\mathrm{g} / \mathrm{cm}^{3}$ )

\begin{tabular}{llll}
\hline theory, author & $\rho_{C}$ & $\rho_{S}$ & $\rho_{M}$ \\
\hline DE405 & 1.8 & 2.4 & 5.0 \\
PitjevaDE & $1.36 \pm 0.03$ & $2.67 \pm 0.03$ & - \\
Pitjeva $_{E P M}$ & $1.36 \pm 0.03$ & $2.67 \pm 0.03$ & - \\
Standish $_{2000}$ & $1.29 \pm 0.06$ & $2.71 \pm 0.04$ & $5.29 \pm 0.53$ \\
\hline
\end{tabular}

\section{Conclusions}

Thus, the considerable progress in the determination of the parameters of planetary ephemerides using radiometric observations is due to the improvement of reduction techniques and dynamical models and also to the improvement of quality and increase of quantity of observational data. Further improvement of the accuracy of planetary parameters will depend on additional precise observations (it is desirable to obtain measurements of landers) and on the significant progress in the determination of asteroid masses, which, as was shown in the paper by Standish (2000b), restricts the accuracy of the planetary ephemerides.

Acknowledgements. The author thanks E. M. Standish (JPL) for helpful discussion of the results and improvements of the original manuscript.

\section{References}

Anderson, J. D., Jurgens, R. F., Lau, E. L., Slade, M. A. III, \& Schubert, G. 1996, Icarus, 124, 690

Brown, T. M., Chrestensen-Dalsgaard, J., Dziembowski, W. A., et al. 1989, ApJ, 343, 526

Brumberg, V. A. 1972, Relativistic Celestial Mechanics (Izdatel'stvo Nauka, Moskva, in Russian)

Duvall, T. L. Jr., Dziembowski, W. A., Goode, P. R., et al. 1984, Nature, 310, 22

Folkner, W. M., Yoder, C. F., Yuan, D. N., Standish, E. M., \& Preston, R. A. 1997, Science, 278, 1749
Harmon, J. K., Cambell, D. B., Bindschadler, D. L., Head, J. W., \& Shapiro, I. I. 1985, Lunar and Planet Sci., 16, 318

Konopliv, A. S., \& Sjogren, W. L. 1995, The JPL Mars gravity field, Mars 50c, based upon Viking and Mariner 9 doppler tracking data, Publication Jet Propulsion Laboratory 95-3, California Inst. of Tech., Pasadena, CA

Pijpers, F. P. 1998, MNRAS, 297, L76

Pitjeva, E. V. 1996, in III International workshop on positional astronomy and celestial mechanics, ed. A. Lopez Garcia, E. I. Yagudina, M. J. Martinez Uso, \& A. Cordero Barbero, Valencia, 583

Pitjeva, E. V. 1997, in Proc. IAU Coll.165, Dynamics and astrometry of natural and artificial celestial bodies, ed. I. M. Wytrzyszczak, J. H. Lieske, \& R. A. Feldman (Kluwer Academic Publishers, Dordrecht), 251

Pitjeva, E. V. 1999, Proceedings of the IAA RAS 4, St.-Petersburg, 22, in Russian

Pitjeva, E. V. 2000, Proceedings of the IAA RAS 5, St.-Petersburg, 58, in Russian

Pitjeva, E. V. 2001, Modern numerical ephemerides of planets and the importance of ranging observations for their creation, Celest. Mech., in press

Standish, E. M. Jr., \& Hellings, R. W. 1989, Icarus, 80, 326

Standish, E. M. Jr. 1990, A\&A, 233, 252

Standish, E. M., Newhall XX, Williams, J. G., \& Folkner, W. M. 1995, JPL planetary and lunar ephemerides, DE403/LE403, IOM 314.10-127, Publication Jet Propulsion Laboratory, Pasadena

Standish, E. M. 1998, JPL planetary and lunar ephemerides, DE405/LE405, IOM 312, F-98-048, Publication Jet Propulsion Laboratory, Pasadena

Standish, E. M. 2000a, Recommendation of DE405 for the 2001 Mars Surveyor mission and for Cassini, IOM 312. F-00-107, Publication Jet Propulsion Laboratory, Pasadena

Standish, E. M. Jr. 2000b, in Proc. IAU Coll. 180, Towards models and constants for sub-microarcsecond astrometry, ed. K. J. Johnston, McMarthy D. D., Luzum B. J., \& Kaplan G. H., U.S. Naval Observatory, Washington, 120

Yeomans, D. K., Barriot, J.-P., Dunham, D. W., et al. 1997, Science, 278, 2106

Yeomans, D. K., Antreasian, P. G., Cheng, A., et al. 1999, Science, 285, 560

Yoder, C. F., \& Standish, E. M. 1997, J. Geophys. Res., 102, $\mathrm{E} 2,4065$ 